\title{
A CASE OF THORACIC STOMACH ${ }^{\mathrm{r}}$
}

\author{
PERCIVAL BAILEY
}

Anatomical Laboratory of Northwestern University Medical School

TWO FIGURES

There have been many cases reported of hernia of the stomach into the thorax, but so far as I can find in the literature, no actual case of development of the stomach in the thorax. Such a case I wish to report.

The condition was found in the course of the regular dissection. The body was that of a certain Ephraim Trimble, who died in the Lake Forest Sanitarium of Cook County, at the age of seventy-seven. In his clinical history there was, strange to say, no mention of any gastric disturbance, and the nurse in charge of his ward stated that his appetite was good to within a short time of his death and that he never complained of any trouble referable to his stomach. Neither were there any discoverable cardiac disturbances. Clinically, there was no reason to suspect any abnormality. He died of arteriosclerosis and interstitial nephritis.

On opening the abdomen, it was found that only the pyloric antrum was present in the abdominal cavity, projecting downward a distance of $6 \mathrm{~cm}$. (fig. 1). At the opening in the diaphragm, the diameter of the stomach was about $2 \mathrm{~cm}$. The duodenum was of course practically completely visible, only the lowest part being covered by the transverse mesocolon. For a distance of $10 \mathrm{~cm}$. beyond the pyloric sphincter it was bound to the liver by the lesser omentum. The rest of the duodenum not covered by the transverse mesocolon was attached only by the dorsal meso, resembling in shape very much the sigmoid mesocolon, an unusual case of persistence of the dorsal mesoduodenum.

${ }^{1}$ Contribution no. 75. 
The opening in the diaphragm lay at the level of the eleventh thoracic vertebra. It was $4 \mathrm{~cm}$. in width. The anterior border was formed by a tough fibrous band which was attached at either end to the body of the eleventh thoracic vertebra and hugged the vertebra closely so that. the opening was a semicircular slit. Through it the abdominal cavity communicated freely with a cavity in the thorax.

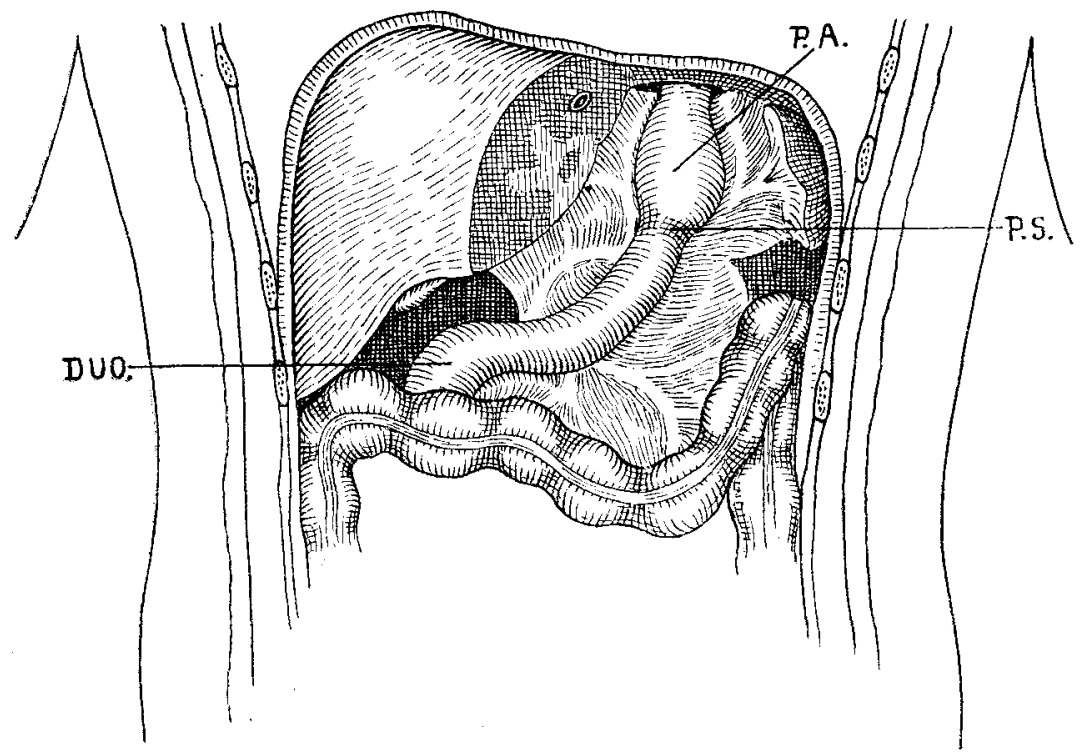

Fig. 1 Anterior view of upper part of abdomen. The left lobe of the liver has been removed. P.A., pyloric antrum; P.S., pyloric sphincter; DUO., duodenum.

On opening the thorax, the body of the stomach was found to lie between the pleural cavities and behind the pericardium, in the posterior mediastinum. It was completely surrounded by a serous sac which was continuous with the peritoneum through the opening in the diaphragm. (Fig. 2). There was a rudimentary greater omentum. The left gastric artery arose from the coeliac and the left gastroepiploic from the splenic at the usual level and passed upward through the diaphragm to the stomach. 
The oesophagus was straight and ended at the level of the third costal cartilage.

It will be seen from this brief description that there is no evidence that the stomach had herniated into the thorax. Its position and relations may be explained by supposing that the anlage of the stomach lay abnormally far anterior on the ali-

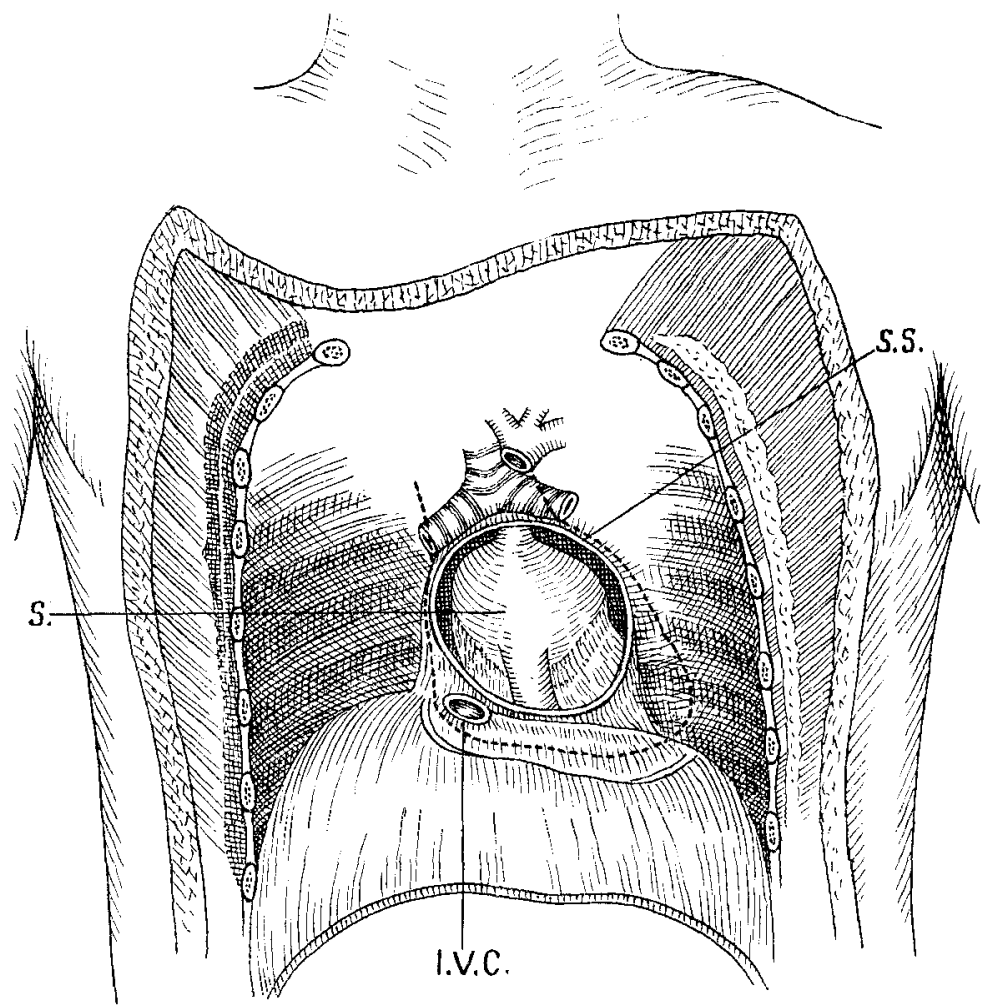

Fig. 2 Anterior view of thorax. Lungs and heart removed and serous sac surrounding stomach opened. Position of heart indicated by dotted lines. $S .$, stomach; S.S., serous sac around stomach; I.V.C., inferior vena cava.

mentary canal. The peritoneal pockets around the stomach develop before the descent of the diaphragm, and finding the peritoneal relations already developed, the diaphragm would necessarily leave above it, as it descended, not only the stomach. but also the extension of the peritoneal cavity. 